\title{
Hetero-Diels-Alder reactions of hetaryl thiochalcones with acetylenic dienophiles
}

\author{
Mlostoń, Grzegorz ; Grzelak, Paulina ; Heimgartner, Heinz
}

\begin{abstract}
Hetaryl-substituted thiochalcones react with acetylenic mono- and diesters in the THF solution in the presence of $\mathrm{LiClO} 4$ at $65^{\circ} \mathrm{C}$ to give, after $24 \mathrm{~h}, 4 \mathrm{H}$-thiopyran carboxylates and dicarboxylates, respectively, in moderate to good yields. The same reactions were performed also in the THF solution without a catalyst under microwave irradiation. In that case, the reaction time was reduced to three minutes and, in most cases, an improvement in the yield of the [4+2]-cycloadduct was observed. The reactions with methyl propiolate occurred regioselectively and the 3-carboxylates were formed exclusively.
\end{abstract}

DOI: https://doi.org/10.1080/17415993.2016.1230857

Posted at the Zurich Open Repository and Archive, University of Zurich ZORA URL: https://doi.org/10.5167/uzh-126307

Journal Article

Accepted Version

Originally published at:

Mlostoń, Grzegorz; Grzelak, Paulina; Heimgartner, Heinz (2017). Hetero-Diels-Alder reactions of hetaryl thiochalcones with acetylenic dienophiles. Journal of Sulfur Chemistry, 38(1):1-10.

DOI: https://doi.org/10.1080/17415993.2016.1230857 


\title{
Hetero-Diels-Alder reactions of hetaryl-thiochalcones with acetylenic dienophiles
}

Grzegorz Mlostoń $^{\mathrm{a}}$, Paulina Grzelak ${ }^{\mathrm{a}}$, and Heinz Heimgartner ${ }^{\mathrm{b}}$

${ }^{a}$ Department of Organic and Applied Chemistry, University of Łódź, Tamka 12, PL-91-403 Łódź, Poland;

${ }^{b}$ Department of Chemistry, University of Zurich, Winterthurerstrasse 190, CH-8057 Zurich, Switzerland

\begin{abstract}
Hetaryl-substituted thiochalcones react with acetylenic mono- and diesters in THF solution in the presence of $\mathrm{LiClO}_{4}$ at $65^{\circ} \mathrm{C}$ to give, after $24 \mathrm{~h}, 4 H$-thiopyran carboxylates and dicarboxylates, respectively, in moderate to good yields. The same reactions were performed also in THF solution without catalyst under microwave irradiation. In that case, the reaction time was reduced to three minutes and, in most cases, an improvement of the yield of the [4+2]-cycloadduct was observed. The reactions with methyl propiolate occurred regioselectively and the 3 -carboxylates were formed exclusively.
\end{abstract}

Graphical Abstract

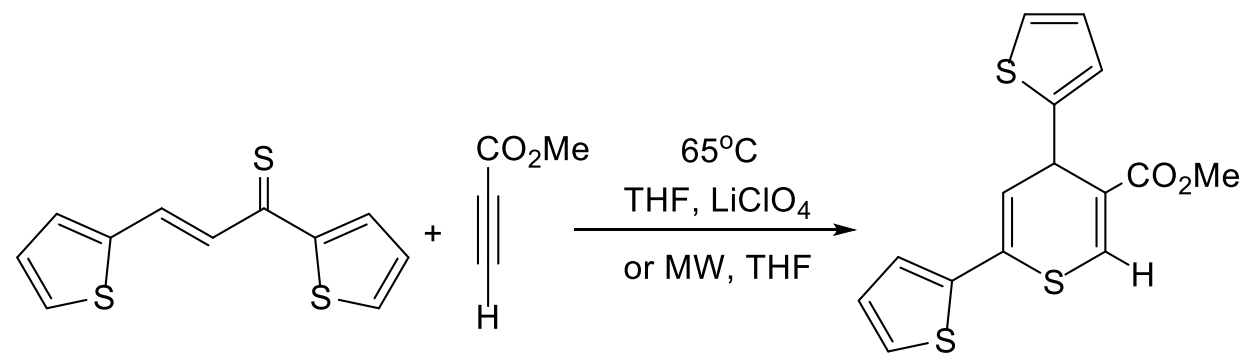

Keywords: chalcones, thiochalcones, acetylenic dienophiles, hetero-Diels-Alder reactions, $4 H$-thiopyrans, microwave irradiation 


\section{Introduction}

Hetero-Diels-Alder reactions of heterodienes are widely applied for the synthesis of sixmembered heterocycles including important intermediates in natural product synthesis $[1,2]$. In that case, the asymmetric version is of special importance [3]. The most frequently reported hetero-Diels-Alder reactions belong to the so-called aza- or oxa-types with the $\mathrm{C}=\mathrm{N}$ or $\mathrm{C}=\mathrm{O}$ units in the diene or dienophile. In contrast, examples for thia-type hetero-Diels-Alder reactions are less well-known. Whereas reactions of dienes with diaryl thioketones leading to dihydrothiopyrans were studied to some extent [4], the [4+2]-cycloadditions with thiochalcones are rarely described [5]. In most cases the studied thia-hetero-Diels-Alder reactions comprised reactions of other types of thia-heterodienes, such as enaminothiones [5] or $\alpha, \beta$-unsaturated thioamides $[5,6]$. In a series of recent publications, 5-arylidene-4-thioxo-2thiazolidinones were used as more complex 1-thia-1,3-dienes in reactions with diverse ethylenic dipolarophiles [7-10]. Furthermore, a few thia-hetero-Diels-Alder reactions of diverse 1-thia-1,3-dienes (but not thiochalcones !) with propiolates [6,7], benzynes [11], and an intramolecular reaction with a propargyl ether [12] are known.

Kinetic studies of the [4+2]-cycloadditions of cyclic and acyclic dienes with diaryl thioketones led to the introduction of the name "superdienophiles" for the latter [4].

An interesting class of S-heterodienes are hetaryl thioketones of type 1, which react with activated ethylene and acetylene derivatives yielding fused thiopyran derivatives of type $\mathbf{2}$ and $\mathbf{3}$, respectively $[13,14]$ (Scheme 1).

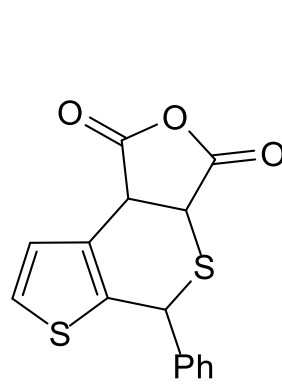

2 [6]

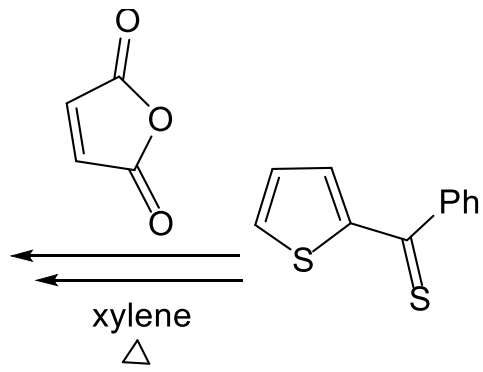

1

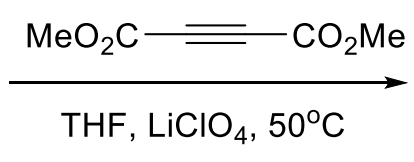

$\mathrm{THF}, \mathrm{LiClO}_{4}, 50^{\circ} \mathrm{C}$<smiles>COC(=O)C1=C(C(=O)OC)c2ccsc2C(c2ccccc2)S1</smiles>

3 [7]

Scheme 1. Hetero-Diels-Alder reactions with hetaryl thioketone 1. 
Thiochalcones 4, which are $\alpha, \beta$-unsaturated thioketones, form a class of attractive thiadienes. They have rarely been explored in hetero-Diels-Alder reactions [5], mainly with ethylenic dienophiles. For example, the reaction of 2,4-diphenyl-1-thiabuta-1,3-diene (4a) with acrylonitrile led to the dihydrothiopyran $\mathbf{5}$ in a regioselective manner [15] (Scheme 2). The reactive thiochalcone $\mathbf{4 a}$ was generated in-situ by thermolysis of its dimer $\mathbf{6}$.<smiles>S=C(c1ccccc1)C1C(c2ccccc2)C=C(c2ccccc2)SC1c1ccccc1</smiles>

6

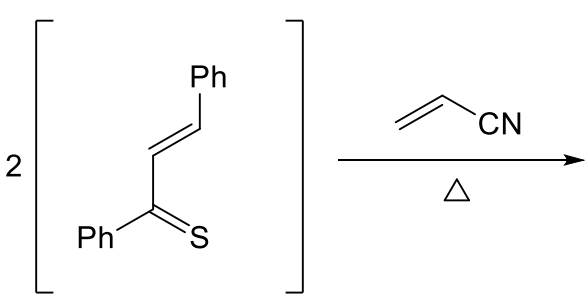

$4 a$<smiles>N#CC1CSC(c2ccccc2)=CC1c1ccccc1</smiles>

5

Scheme 2. [4+2]-Cycloaddition of diphenylchalcone with acrylonitrile.

Remarkably, thiochalcone 4a reacted also with the electron-rich $n$-butyl vinyl ether to give the [4+2]-cycloadduct in a Diels-Alder reaction with inverse electron demand [16]. Furthermore, the reaction of dimethyl acetylene dicarboxylate (DMAD, 8a) with in-situgenerated thiochalcones yielded $4 H$-thiopyrans [17]. In the case of enaminethiones, the reactions with methyl propiolate (MP, 8b) occurred regioselectively leading to 4-amino- $4 \mathrm{H}$ thiopyran-3-carboxylates as primary products, which upon heating isomerized to 2 -amino- $2 \mathrm{H}$ thiopyran-3-carboxylates $[18,19]$.

Due to our continuing interest in the chemistry of hetaryl thioketones, a series of new thiochalcones with hetaryl groups was prepared and subsequently applied in hetero-DielsAlder reactions with DMAD and MP.

\section{Results and discussion}

Hetaryl thiochalcones $\mathbf{4 b}-\mathbf{g}$ (Table 1) were prepared from the corresponding chalcones by treatment with Lawesson's reagent in refluxing THF solution and isolated after chromatographic workup. The ${ }^{1} \mathrm{H}$ NMR spectra of analytically pure samples showed that they exist in $\mathrm{CDCl}_{3}$ solution in equilibrium with dimeric forms similar to the observations with diarylchalcones [20] (Scheme 3). 
<smiles>[Al]C(=S)C1C(Br)C=C([Al])SC1Br</smiles>

6<smiles>S=C(Br)/C=C/Br</smiles>

4<smiles>[18F]C1(C=C[Al])SSC([123I])=CC1Br</smiles>

7

Scheme 3. Equilibrium of thiochalcones with dimers.

Nevertheless, the cycloaddition reactions performed with acetylenic dienophiles $\mathbf{8}$ occur smoothly in THF solution in the presence of catalytic amounts of $\mathrm{LiClO}_{4}$. After $24 \mathrm{~h}$ of heating at $65^{\circ} \mathrm{C}$ in a glass tube with a screw cap, the reactions were complete and the ${ }^{1} \mathrm{H}$ NMR analysis evidenced the presence of a single product in all cases. The spectroscopic data confirmed the structure of $4 H$-thiopyrane derivatives 9 (Scheme 4, Table 1). Thus, in the case of the products formed from $\operatorname{DMAD}(\mathbf{8 a})$ and $\mathbf{4 b}$ and $\mathbf{4 d}$, respectively, two isomeric compounds were identified. In the first one, 9b, the ${ }^{1} \mathrm{H}$ NMR spectrum showed two characteristic doublets at 6.24 and $5.27 \mathrm{ppm}$ with $J=6.6 \mathrm{~Hz}$ attributed to $\mathrm{HC}(5)$ and $\mathrm{HC}(4)$ of the thiopyran ring. The analogous set of signals in the spectrum of $9 \mathbf{d}$ appeared at 6.22 and $4.85 \mathrm{ppm}(J=6.6 \mathrm{~Hz})$. In the ${ }^{13} \mathrm{C}$ NMR spectra, a significant difference in the chemical shift of HC(4) was observed. Whereas in 9d the signal appeared at $44.5 \mathrm{ppm}$, it was shifted upfield to $38.6 \mathrm{ppm}$ in $\mathbf{9 b}$.

The structure of the products obtained regioselectively in the experiments with methyl propiolate (8b) were unambiguously established on the basis of the ${ }^{1} \mathrm{H}$ NMR spectra. In all compounds $\mathbf{9 h - n}$, the signals of $\mathrm{HC}(4)$ appeared as a doublet in the range of 4.81-5.26 ppm. In addition, the singlet for $\mathrm{HC}(2)$ was located in a narrow range at 7.67-7.90 ppm. The same regioselectivity was observed in the reaction of the parent thiochalcone $\mathbf{4 a}$ with $\mathbf{8 b}$, and the structure 9h was elucidated spectroscopically. The comparison of the chemical shifts observed in the ${ }^{13} \mathrm{C}$ NMR spectra of $9 \mathbf{h}$ and $9 \mathbf{i}$ evidences that the shielding effect of the thiophen-2-yl substituent is stronger than in the case of the phenyl ring.

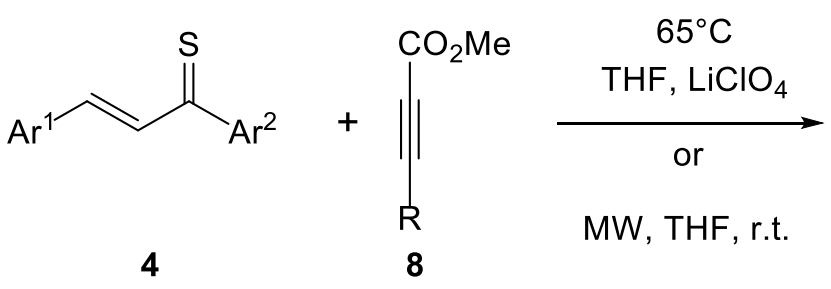<smiles>[Z17]C1=CC(Br)C(C(C)=O)=C([R])S1</smiles> 
Scheme 4. Hetero-Diels-Alder reactions of thiochalcones 4 with acetylene carboxylates.

Table 1. Synthesis of $4 H$-thiopyrans 9 via hetero-Diels-Alder reaction

\begin{tabular}{|c|c|c|c|c|c|c|}
\hline \multirow{2}{*}{$\begin{array}{l}\text { Acetylene } \\
8 \mathrm{R}\end{array}$} & \multicolumn{3}{|c|}{ Thiochalcone $^{\mathrm{a}}$ ) } & \multirow{2}{*}{$\begin{array}{c}\text { Thiopyran } \\
9\end{array}$} & \multicolumn{2}{|c|}{ Yield $\left.[\%]^{b}\right)$} \\
\hline & 4 & $\operatorname{Ar}^{1}$ & $\mathrm{Ar}^{2}$ & & Method A & Method B \\
\hline a $\mathrm{CO}_{2} \mathrm{Me}$ & $\mathbf{a}$ & $\mathrm{Ph}$ & $\mathrm{Ph}$ & $\mathbf{a}[10]$ & 80 & 93 \\
\hline a $\mathrm{CO}_{2} \mathrm{Me}$ & b & Thi & $\mathrm{Ph}$ & b & 85 & 86 \\
\hline a $\mathrm{CO}_{2} \mathrm{Me}$ & c & Fur & $\mathrm{Ph}$ & c & 77 & 95 \\
\hline a $\mathrm{CO}_{2} \mathrm{Me}$ & d & $\mathrm{Ph}$ & Thi & d & 36 & 48 \\
\hline a $\mathrm{CO}_{2} \mathrm{Me}$ & e & $\mathrm{Ph}$ & Fur & e & 6 & c) \\
\hline a $\mathrm{CO}_{2} \mathrm{Me}$ & f & $\mathrm{Ph}$ & Sel & f & 16 & 56 \\
\hline a $\mathrm{CO}_{2} \mathrm{Me}$ & g & Thi & Thi & g & 67 & d) \\
\hline b $\mathrm{H}$ & $\mathbf{a}$ & $\mathrm{Ph}$ & $\mathrm{Ph}$ & $\mathbf{h}[10]$ & 85 & 93 \\
\hline b $\mathrm{H}$ & b & Thi & $\mathrm{Ph}$ & i & 92 & 92 \\
\hline b $\mathrm{H}$ & c & Fur & $\mathrm{Ph}$ & $\mathbf{j}$ & 94 & 98 \\
\hline b $\mathrm{H}$ & d & $\mathrm{Ph}$ & Thi & $\mathbf{k}$ & 48 & 71 \\
\hline b $\mathrm{H}$ & e & $\mathrm{Ph}$ & Fur & 1 & 6 & c) \\
\hline b $\mathrm{H}$ & f & $\mathrm{Ph}$ & Sel & $\mathbf{m}$ & 15 & 60 \\
\hline b $\mathrm{H}$ & g & Thi & Thi & $\mathbf{n}$ & 97 & d) \\
\hline
\end{tabular}

${ }^{\text {a) }} \mathrm{Ph}=$ Phenyl; Thi = Thiophen-2-yl; Fur = Furan-2-yl; Sel = Selenophen-2-yl

b) Yield of isolated product. Method A: THF, $\mathrm{LiClO}_{4}, 65^{\circ} \mathrm{C}$; Method B: THF, MW, r.t.

$\left.{ }^{c}\right)$ Only decomposition products were obtained

d) Experiment has not been performed 
The microwave technique has been widely applied to accelerate organic reactions. In the present study, the reactions of DMAD (8a) and PM (8b) with hetaryl thiochalcones 4 were carried out in THF solutions under microwave irradiations (Method B). As a matter of fact, the reaction times were drastically reduced (ca. $3 \mathrm{~min}$ ) and the yields of the products were higher (Table 1). Only in the case of thiochalcone $4 \mathbf{e}$ with the furan-2-yl group at $C(1)$, decomposition was observed, similarly to the reaction performed under standard conditions.

\section{Conclusions}

Hetaryl thiochalcones bearing the hetaryl group either at $C(2)$ or $C(4)$ of the 1-thiabuta-1,3diene structure react smoothly with acetylene carboxylates yielding the expected $4 \mathrm{H}$ thiopyran derivatives. In the case of methyl propiolate (MP), the [4+2]-cycloaddition occurs regioselectively yielding thiopyran-3-carboxylates. Whereas thermal reactions performed at $65^{\circ} \mathrm{C}$ in the presence of $\mathrm{LiClO}_{4}$ as a catalyst required ca. $24 \mathrm{~h}$ for completion, the noncatalyzed microwave-supported reactions were finished after ca. 3 min.

\section{Experimental Design}

\subsection{General}

Melting points were determined in a capillary using a MEL-TEMP II apparatus (Aldrich) and are uncorrected. IR spectra were recorded with a FT-IR NEXUS spectrophotometer as $\mathrm{KBr}$ pellets; absorptions $(v)$ in $\mathrm{cm}^{-1} .{ }^{1} \mathrm{H}$ and ${ }^{13} \mathrm{C}$ NMR spectra were measured on a Bruker Avance III $\left({ }^{1} \mathrm{H}\right.$ at 600 and ${ }^{13} \mathrm{C}$ at $\left.150 \mathrm{MHz}\right)$ instrument in $\mathrm{CDCl}_{3}$; chemical shifts $(\delta)$ are given in ppm, coupling constants $(J)$ in Hz. The multiplicity of the ${ }^{13} \mathrm{C}$ signals was deduced from DEPT, supported by ${ }^{1} \mathrm{H}_{-}{ }^{13} \mathrm{C}$ HMQC spectra. ${ }^{1} \mathrm{H}$ NMR data are presented as follows: chemical shift, multiplicity $(\mathrm{br}=$ broad, $\mathrm{s}=$ singlet, $\mathrm{d}=$ doublet, $\mathrm{t}=$ triplet, $\mathrm{q}=$ quartet, $\mathrm{m}=$ multiplet), coupling constant, integration. The mass spectra were recorded on a Finnigan MAT-95(ESI), Bruker maxis (HR-ESI), or SYNAPT G2-S HMDS (HR MALDI-TOF) instrument. Elemental analyses were performed in the Microanalytical Laboratory of the Chemistry Faculty in Łódź. Microwave experiments were carried out with CEM Focused Microwave type Discover SPD. Applied reagents such as dimethyl acetylenedicarboxylate (DMAD, 8a), methyl propiolate (MP, 8b), inorganic reagents, and solvents are commercially available (Aldrich) and were used as received. 


\section{2. $\quad$ Starting materials}

Chalcones were obtained from aryl methyl ketones and aromatic aldehydes in the presence of $\mathrm{NaOH}$ according to the known general procedure [21]. 1,3-Diphenylpropen-3-one: Pale yellow crystals; yield: 91\%; m.p. $55.5-56.0^{\circ} \mathrm{C}\left(\mathrm{C}_{2} \mathrm{H}_{5} \mathrm{OH}\right)$ ([22]: m.p. $\left.56.0^{\circ} \mathrm{C}\right)$. 1-Phenyl-3(thiophen-2-yl)prop-2-en-1-one: Yellow crystals; yield: $84 \%$; m.p. $58.0-59.0^{\circ} \mathrm{C}\left(\mathrm{C}_{2} \mathrm{H}_{5} \mathrm{OH}\right)$ ([23]: m.p. $58.0-59.0^{\circ} \mathrm{C}$ ). 3-Phenyl-1-(thiophen-2-yl)prop-2-en-1-one: Pale yellow crystals; yield: $78 \%$; m.p. $82.5-83.0^{\circ} \mathrm{C}\left(\mathrm{C}_{2} \mathrm{H}_{5} \mathrm{OH}\right)$ ([24], m.p. $\left.82^{\circ} \mathrm{C}\right)$. 3-(Furan-2-yl)-1-phenylprop-2en-1-one: Pale yellow crystals; yield: $85 \%$; m.p. $42.0-42.5^{\circ} \mathrm{C}\left(\mathrm{C}_{2} \mathrm{H}_{5} \mathrm{OH}\right)\left([25]\right.$, m.p. $\left.40.0^{\circ} \mathrm{C}\right)$. 1-(Furan-2-yl)-3-phenylprop-2-en-1-one: Pale yellow crystals; yield: 71\%; m.p. 86.0-87.0 ${ }^{\circ} \mathrm{C}$ $\left(\mathrm{C}_{2} \mathrm{H}_{5} \mathrm{OH}\right)$ ([26]: m.p. 87.0-88.0 $\left.{ }^{\circ} \mathrm{C}\right)$. 3-Phenyl-1-(selenophen-2-yl)prop-2-en-1-one: Pale yellow crystals; yield: $82 \%$; m.p. $81.5-82.0^{\circ} \mathrm{C}\left(\mathrm{C}_{2} \mathrm{H}_{5} \mathrm{OH}\right)$ ([27]: m.p. 81.0-82.5 $\left.{ }^{\circ} \mathrm{C}\right)$. 1,3Di(thiophen-2-yl)prop-2-en-1-one: Yellow crystals; yield: 70\%; m.p.95.5-96.0 ${ }^{\circ} \mathrm{C}\left(\mathrm{C}_{2} \mathrm{H}_{5} \mathrm{OH}\right)$ ([25]: m.p.94-95 $\left.{ }^{\circ} \mathrm{C}\right)$.

\subsection{Synthesis of hetaryl thiochalcones $(4 a-g)$}

A solution of $5 \mathrm{mmol}$ of the corresponding chalcone and $5.1 \mathrm{mmol}$ of Lawesson's reagent in $10 \mathrm{~mL}$ of dry THF were placed in a two-necked flask. The mixture was heated under argon at reflux for $3 \mathrm{~h}$. Then, the solvent was evaporated in vacuo and the residue was purified chromatographically, using as the eluent a mixture of petroleum ether and chloroform (1:1). The ${ }^{1} \mathrm{H}$ NMR spectra showed the presence of variable mixtures of monomers and dimers of the thiocalcones 4 .

\section{1,3-Diphenylpropen-3-thione (4a)}

Purple solid; yield: $672 \mathrm{mg}$ (65\%); m.p. 129.0-130.0 ${ }^{\circ} \mathrm{C}$ (lit. [15]: m.p. 134.0-135.0 ${ }^{\circ} \mathrm{C}$ ). Anal. calcd for $\mathrm{C}_{15} \mathrm{H}_{12} \mathrm{~S}$ (224.33): C 80.30, H 5.40, S 14.29; found: C 80.14, H 5.64, S 14.37. 
Purple solid; yield: $862.5 \mathrm{mg}(75 \%)$; m.p. $60.5-61.0^{\circ} \mathrm{C}$. Anal. calcd for $\mathrm{C}_{13} \mathrm{H}_{10} \mathrm{~S}_{2}$ (230.35): C 67.78, H 4.38, S 27.83; found: C 67.69 , H 4.54, S 27.84.

3-(Furan-2-yl)-1-phenylprop-2-en-1-thione (4c)

Purple solid; yield: $770.4 \mathrm{mg}$ (72\%); m.p. 59.0-59.5 ${ }^{\circ} \mathrm{C}$. Anal. calcd for $\mathrm{C}_{13} \mathrm{H}_{10} \mathrm{OS}$ (214.29): C 72.86, H 4.71, S 14.96; found: C 72.76, H 4.71, S 14.81.

3-Phenyl-1-(thiophen-2-yl)prop-2-en-1-thione (4d)

Green solid; yield: $931.5 \mathrm{mg}$ (81\%); m.p. $76.0-76.5^{\circ} \mathrm{C}$. Anal. calcd for $\mathrm{C}_{13} \mathrm{H}_{10} \mathrm{~S}_{2}$ (230.35): C 67.78, H 4.38, S 27.83; found: C 67.59, H 4.56, S 27.73.

1-(Furan-2-yl)-3-phenylprop-2-en-1-thione (4e)

Green solid; yield: $802.5 \mathrm{mg}(75 \%)$; m.p. $110.0-110.5^{\circ} \mathrm{C}$. Anal. calcd for $\mathrm{C}_{13} \mathrm{H}_{10} \mathrm{OS}$ (214.29): C 72.86, H 4.71, S 14.96; found: C 72.86, H 4.97, S 14.70.

3-Phenyl-1-(selenophen-2-yl)prop-2-en-1-thione (4f)

Green solid; yield: $844.5 \mathrm{mg}$ (61\%); m.p. $115.0-115.5^{\circ} \mathrm{C}$. Anal. calcd for $\mathrm{C}_{13} \mathrm{H}_{10} \mathrm{SeS}$ (277.25): C 56.31, H 3.64, S 11.56; found: C 56.16, H 3.89, S 11.58.

\section{1,3-Di(thiophen-2-yl)prop-2-en-1-thione (4g)}

Green solid; yield: $840 \mathrm{mg}$ (70\%); m.p. $80.0-80.5^{\circ} \mathrm{C}$. Anal. calcd for $\mathrm{C}_{11} \mathrm{H}_{8} \mathrm{~S}_{3}$ (236.37): C 55.89, H 3.42, S 40.69; found: C 55.73, H 3.67, S 40.53.

\subsection{Reaction of thiochalcones 4 with acetylenic dienophiles 8 -General procedures}

Method A: A solution of $1 \mathrm{mmol}$ of the corresponding thiochalcone 4, $1.1 \mathrm{mmol}$ of the corresponding acetylenic dienophile 8 and $10 \mathrm{~mol} \%$ of $\mathrm{LiClO}_{4}$ in $1 \mathrm{~mL}$ of dry THF were placed in a thick-wall glass tube, which then was closed with a scrue cap. The mixture was 
heated at $65^{\circ} \mathrm{C}$ for $24 \mathrm{~h}$. The solvent was evaporated in vacuo. The residue was purified chromatographically, using as the eluent a mixture of petroleum ether and ethyl acetate (1:1).

Method B: A solution of $1 \mathrm{mmol}$ of the corresponding thiochalcone and $1.1 \mathrm{mmol}$ of the corresponding acetylenic dienophile 8 in $1 \mathrm{~mL}$ of dry THF were placed in a reaction tube. The reaction tube was sealed and transferred to the microwave reactor Discover SPD (150 W, 3 min). After cooling the reaction mixture to room temperature, the solvent was evaporated in vacuo. The residue was purified chromatographically, using as the eluent a mixture of petroleum ether and ethyl acetate (1:1).

Dimethyl 4, 6-diphenyl-4H-thiopyran-2,3-dicarboxylate (9a) [17]

Yellow oil; yield (method B): $340.4 \mathrm{mg}(93 \%) .{ }^{1} \mathrm{H} \mathrm{NMR}\left(600 \mathrm{MHz}, \mathrm{CDCl}_{3}\right): 3.58,3.77$ $\left(2 s, 6 \mathrm{H}, 2 \mathrm{OCH}_{3}\right), 4.82(d, J=6.6 \mathrm{~Hz}, 1 \mathrm{H}), 6.06(d, J=6.6 \mathrm{~Hz}, 1 \mathrm{H}), 7.16-7.18(m, 1 \mathrm{H}), 7.23-$ $7.26(m, 5 \mathrm{H}), 7.29-7.31(m, 2 \mathrm{H}), 7.38-7.39(m, 2 \mathrm{H})$.

Dimethyl 6-phenyl-4-(thiophen-2-yl)-4H-thiopyran-2,3-dicarboxylate (9b)

Orange oil; yield (method B): $319.9 \mathrm{mg}(86 \%) .{ }^{1} \mathrm{H} \mathrm{NMR}\left(600 \mathrm{MHz}, \mathrm{CDCl}_{3}\right): 3.77,3.87$ $\left(2 s, 6 \mathrm{H}, 2 \mathrm{OCH}_{3}\right), 5.27(d, J=6.6 \mathrm{~Hz}, 1 \mathrm{H}), 6.24(d, J=6.6 \mathrm{~Hz}, 1 \mathrm{H}), 6.94-6.95(\mathrm{~m}, 1 \mathrm{H}), 7.01$ $(d, J=3.6 \mathrm{~Hz}, 1 \mathrm{H}), 7.20(d, J=4.8 \mathrm{~Hz}, 1 \mathrm{H}), 7.36-7.38(m, 3 \mathrm{H}), 7.51-7.52(m, 2 \mathrm{H}) .{ }^{13} \mathrm{C} \mathrm{NMR}$ (150 MHz, $\left.\mathrm{CDCl}_{3}\right): 38.6(\mathrm{C}(4))$, 52.6, $53.2\left(2 \mathrm{OCH}_{3}\right), 126.1,132.6,135.5,136.5,144.1$ $\left(5 \mathrm{C}\left(\mathrm{sp}^{2}\right)\right), 119.9,125.1,126.9,127.0,128.8,129.2\left(9 \mathrm{CH}\left(\mathrm{sp}^{2}\right)\right), 165.2,165.4(2 \mathrm{C}=\mathrm{O}) . \mathrm{IR}$ (film): 3025w, 2952w, 1736, 1715vs $\left(2 v_{\mathrm{C}=\mathrm{O}}\right), 1597 w, 1492 w, 1442 w, 1337 w, 1261 s, 1021 w$, 761w, 694m. Anal. calcd for $\mathrm{C}_{19} \mathrm{H}_{16} \mathrm{O}_{4} \mathrm{~S}_{2}$ (372.47): C 61.26, H 4.34, S 17.21; found: $\mathrm{C}$ 61.12, H 4.5, S 17.14 .

Dimethyl 4-(furan-2-yl)-6-phenyl-4H-thiopyran-2,3-dicarboxylate (9c)

Orange oil; yield (method B): $338.2 \mathrm{mg}(95 \%) .{ }^{1} \mathrm{H}$ NMR (600 MHz, $\left.\mathrm{CDCl}_{3}\right): 3.77,3.88$ $\left(2 s, 6 \mathrm{H}, 2 \mathrm{OCH}_{3}\right), 5.12(d, J=6.6 \mathrm{~Hz}, 1 \mathrm{H}), 6.17-6.19(m, 2 \mathrm{H}), 6.29-6.30(m, 1 \mathrm{H}), 7.35-7.37$ $(m, 4 \mathrm{H}), 7.49-7.51(m, 2 \mathrm{H}) .{ }^{13} \mathrm{C} \mathrm{NMR}\left(150 \mathrm{MHz}, \mathrm{CDCl}_{3}\right): 37.9(\mathrm{C}(4)), 52.7,53.3\left(2 \mathrm{OCH}_{3}\right)$, 124.6, $133.7136 .4,136.6,152.6\left(5 \mathrm{C}\left(\mathrm{sp}^{2}\right)\right), 106.7,110.5,117.5,126.9,128.8,129.2,142.5$ $\left(9 \mathrm{CH}\left(\mathrm{sp}^{2}\right)\right), 165.2,165.6(2 \mathrm{C}=\mathrm{O})$. IR (film): 3056w, 2949w, 1733, 1714vs $\left(2 v_{\mathrm{C}=\mathrm{O}}\right), 1594 w$, 
$1492 w, 1442 w, 1255 s, 1011 m, 764 m, 698 w$. Anal. calcd for $\mathrm{C}_{19} \mathrm{H}_{16} \mathrm{O}_{5} \mathrm{~S}$ (356.41): C 64.02, $\mathrm{H}$ 4.53, S 8.99; found: C 63.87, H 4.65, S 8.85.

Dimethyl 4-phenyl-6-(thiophen-2-yl)-4H-thiopyran-2,3-dicarboxylate (9d)

Orange oil; yield (method B): $178.6 \mathrm{mg}(48 \%) .{ }^{1} \mathrm{H} \mathrm{NMR}\left(600 \mathrm{MHz}, \mathrm{CDCl}_{3}\right): 3.66,3.86$ $\left(2 s, 6 \mathrm{H}, 2 \mathrm{OCH}_{3}\right), 4.85(d, J=6.6 \mathrm{~Hz}, 1 \mathrm{H}), 6.22(d, J=6 \mathrm{~Hz}, 1 \mathrm{H}), 6.99(d d, J=4.8,3.6 \mathrm{~Hz}$, 1H), 7.18-7.19 (m, 1H), 7.23-7.27 (m, 2H), 7.30-7.36 (m, 4H). ${ }^{13} \mathrm{C}$ NMR (150 MHz, $\left.\mathrm{CDCl}_{3}\right)$ : 44.5 (C(4)); 52.6, $55.3\left(2 \mathrm{OCH}_{3}\right), 125.0,129.1,133.0,139.8,141.3\left(5 \mathrm{C}\left(\mathrm{sp}^{2}\right)\right), 119.7,125.2$, $125,8,127.7,127.8,128.1,128.8\left(9 \mathrm{CH}\left(\mathrm{sp}^{2}\right)\right), 166.2,164.9(2 \mathrm{C}=\mathrm{O})$. IR (film): $3028 w, 2952 w$, $1730,1721 v s\left(2 v_{\mathrm{C}=0}\right), 1597 w, 1451 w, 1429 m, 1258 s, 1023 m, 761 w, 698 m$. Anal. calcd for $\mathrm{C}_{19} \mathrm{H}_{16} \mathrm{O}_{4} \mathrm{~S}_{2}$ (372.47): C 61.26, H 4.34, S 17.21; found: C 61.43, H 4.58, S 17.19.

Dimethyl 6-(furan-2-yl)-4-phenyl-4H-thiopyran-2,3-dicarboxylate (9e)

Yield (method A): 6\%. The compound was identified only on the basis of the ${ }^{1} \mathrm{H}$ NMR spectra and could not be isolated in pure form. The yield was calculated based on the ${ }^{1} \mathrm{H}$ NMR spectra of the crude mixture using a weighed amount of 1,1,2,2-tetrachloroethane as a standard. ${ }^{1} \mathrm{H}$ NMR (600 MHz, $\left.\mathrm{CDCl}_{3}\right)$ : 3.86, $3.66\left(2 s, 6 \mathrm{H}, 2 \mathrm{OCH}_{3}\right), 4.85(d, J=6.6 \mathrm{~Hz}, 1 \mathrm{H})$, 6.40-6.41 (m, 2H), $6.46(d, J=3.0 \mathrm{~Hz}, 1 \mathrm{H}), 7.28-7.33(m, 3 \mathrm{H}), 7.35-7.37(m, 3 \mathrm{H})$.

Dimethyl 4-phenyl-6-(selenophen-2-yl)-4H-thiopyran-2,3-dicarboxylate (9f)

Orange oil; yield (method B): $234.5 \mathrm{mg}(56 \%) .{ }^{1} \mathrm{H}$ NMR (600 MHz, $\left.\mathrm{CDCl}_{3}\right): 3.67,3.86$ $\left(2 s, 6 \mathrm{H}, 2 \mathrm{OCH}_{3}\right), 4.84(d, J=6.6 \mathrm{~Hz}, 1 \mathrm{H}), 6.18(d, J=6.6 \mathrm{~Hz}, 1 \mathrm{H}), 7.22-7.27(m, 2 \mathrm{H}), 7.31-$ $7.34(m, 2 \mathrm{H}), 7.36-7.37(m, 3 \mathrm{H}), 7.92(d, J=5.4 \mathrm{~Hz}, 1 \mathrm{H}) .{ }^{13} \mathrm{C} \mathrm{NMR}\left(150 \mathrm{MHz}, \mathrm{CDCl}_{3}\right): 44.7$ (C(4)), 52.6, $55.3\left(2 \mathrm{OCH}_{3}\right), 127.0,128.8,133.2,141.2,144.6\left(5 \mathrm{C}\left(\mathrm{sp}^{2}\right)\right), 120.3,127.4,127.8$, 128.2, 129.1, 130.2, $131.1\left(9 \mathrm{CH}\left(\mathrm{sp}^{2}\right)\right), 164.9,166.2(2 \mathrm{C}=\mathrm{O}) . \mathrm{IR}$ (film): 3025w, 2952w, 1736, $1726 v s\left(2 v_{\mathrm{C}=0}\right), 1600 w, 1454 w, 1435 m, 1261 s, 1021 w, 786 w, 697 m$. Anal. calcd for $\mathrm{C}_{19} \mathrm{H}_{16} \mathrm{SeO}_{4} \mathrm{~S}$ (419.37): C 54.41, H 3.85, S 7.64; found: C 54.67, H 3.86, S 7.43.

Dimethyl 4,6-bis(thiophen-2-yl)-4H-thiopyran-2,3-dicarboxylate (9g) 
Yield (method A): $253.2 \mathrm{mg}(67 \%)$. The compound was identified only on the basis of the ${ }^{1} \mathrm{H}$ NMR spectra and could not be isolated in pure form. The yield was calculated based on the ${ }^{1} \mathrm{H}$ NMR spectra of crude mixture using a weighed amount of 1,1,2,2-tetrachloroethane as a standard. ${ }^{1} \mathrm{H}$ NMR (600 MHz, $\left.\mathrm{CDCl}_{3}\right): 3.77,3.86\left(2 s, 6 \mathrm{H}, 2 \mathrm{OCH}_{3}\right), 5.20(d, J=20.4 \mathrm{~Hz}$, $1 \mathrm{H}), 6.30(d, J=20.4 \mathrm{~Hz}, 1 \mathrm{H}), 6.91-7.04(m, 3 \mathrm{H}), 7.17-7.30(m, 3 \mathrm{H})$.

Methyl 4,6-diphenyl-4H-thiopyran-3-carboxylate (9h)

Yellow solid; yield (method B): $286.4 \mathrm{mg}$ (93\%); m.p. 76.2-76.7 ${ }^{\circ} \mathrm{C}$ (chromatographic purification). ${ }^{1} \mathrm{H} \mathrm{NMR}\left(600 \mathrm{MHz}, \mathrm{CDCl}_{3}\right): 3.61\left(s, 3 \mathrm{H}, \mathrm{OCH}_{3}\right), 4.81(d, J=6 \mathrm{~Hz}, 1 \mathrm{H}), 6.10$ $(d, J=6.6 \mathrm{~Hz}, 1 \mathrm{H}), 7.12-7.16(m, 1 \mathrm{H}), 7.21-7.27(m, 5 \mathrm{H}), 7.33-7.37(m, 4 \mathrm{H}), 7.67(s, 1 \mathrm{H})$. ${ }^{13} \mathrm{C} \mathrm{NMR}\left(150 \mathrm{MHz}, \mathrm{CDCl}_{3}\right): 41.5(\mathrm{C}(4)), 52.0\left(\mathrm{OCH}_{3}\right), 123.9,129.2,137.7,144.2\left(4 \mathrm{C}\left(\mathrm{sp}^{2}\right)\right)$, $122.3,126.5,127.2,128.1,128.77,128.81,128.84,132.8\left(12 \mathrm{CH}\left(\mathrm{sp}^{2}\right)\right), 165.2(\mathrm{C}=\mathrm{O}) . \mathrm{IR}$ (KBr): $3025 w, 2955 w, 1708 v s\left(v_{\mathrm{C}=0}\right), 1594 w, 1429 m, 1249 s, 1046 w, 755 m, 694 m$. Anal. calcd for $\mathrm{C}_{19} \mathrm{H}_{16} \mathrm{O}_{2} \mathrm{~S}$ (308.41): C 73.99, H 5.24, S 10.39; found: C 74.06, H 5.47, S 10.49.

Methyl 6-phenyl-4-(thiophen-2-yl)-4H-thiopyran-3-carboxylate (9i)

Orange oil; yield (method B): $288.9 \mathrm{mg}(92 \%) .{ }^{1} \mathrm{H} \mathrm{NMR}\left(600 \mathrm{MHz}, \mathrm{CDCl}_{3}\right): 3.78(\mathrm{~s}, 3 \mathrm{H}$, $\left.\mathrm{OCH}_{3}\right), 5.28(d, J=6.6 \mathrm{~Hz}, 1 \mathrm{H}), 6.27(d, J=6.6 \mathrm{~Hz}, 1 \mathrm{H}), 6.95(d d, J=5.4,3.6 \mathrm{~Hz} 1 \mathrm{H}), 7.02$ $(d, J=3.6 \mathrm{~Hz}, 1 \mathrm{H}), 7.18(d, J=5.4 \mathrm{~Hz}, 1 \mathrm{H}), 7.36-7.39(m, 3 \mathrm{H}), 7.51-7.52(m, 2 \mathrm{H}), 7.74(s$, 1H). ${ }^{13} \mathrm{C}$ NMR (150 MHz, $\left.\mathrm{CDCl}_{3}\right): 35.9(\mathrm{C}(4)), 52.0\left(\mathrm{OCH}_{3}\right), 123.6,131.1,137.3,146.8$ $\left(4 \mathrm{C}\left(\mathrm{sp}^{2}\right)\right), 120.9,124.6,126.7,126.9,128.8,128.9,132.9\left(10 \mathrm{CH}\left(\mathrm{sp}^{2}\right)\right), 164.9(\mathrm{C}=\mathrm{O}) . \mathrm{IR}$ (film): 3060w, 2949w, 1708vs ( $\left.v_{\mathrm{C}=\mathrm{O}}\right), 1581 \mathrm{~m}, 1432 \mathrm{~m}, 1236 \mathrm{~s}, 1036 \mathrm{~m}, 761 w, 694 m$. Anal. calcd for $\mathrm{C}_{17} \mathrm{H}_{14} \mathrm{O}_{2} \mathrm{~S}_{2}$ (314.43): C 64.93, H 4.50, S 20.39; found: C 64.83, H 4.73, S 20.47.

Methyl 4-(furan-2-yl)-6-phenyl-4H-thiopyran-3-carboxylate (9j)

Orange solid; yield (method B): $292.0 \mathrm{mg}(98 \%)$; m.p. $75.5-76.0^{\circ} \mathrm{C}$ (chromatographic purification). ${ }^{1} \mathrm{H} \mathrm{NMR}\left(600 \mathrm{MHz}, \mathrm{CDCl}_{3}\right): 3.78\left(s, 3 \mathrm{H}, \mathrm{OCH}_{3}\right), 5.12(d, J=6.6 \mathrm{~Hz}, 1 \mathrm{H}), 6.12$ (br $s, 1 \mathrm{H}), 6.20(d, J=6.6 \mathrm{~Hz}, 1 \mathrm{H}), 6.29$ (br $s, 1 \mathrm{H}), 7.34-7.37(m, 4 \mathrm{H}), 7.49(d, J=7.2 \mathrm{~Hz}$, 2H), $7.79(s, 1 \mathrm{H}) .{ }^{13} \mathrm{C}$ NMR (150 MHz, $\left.\mathrm{CDCl}_{3}\right): 35.0(\mathrm{C}(4)), 52.2\left(\mathrm{OCH}_{3}\right), 121.3,131.9$, 137.4, $154.9\left(4 \mathrm{C}\left(\mathrm{sp}^{2}\right)\right), 106.0,110.5,118.6,126.6,128.8,129.0,134.2,142.0\left(10 \mathrm{CH}\left(\mathrm{sp}^{2}\right)\right)$, $165.0(\mathrm{C}=\mathrm{O})$. IR (KBr): 3044w, 2942w, 1704vs $\left(v_{\mathrm{C}=\mathrm{O}}\right), 1591 w, 1432 w, 1242 s, 1033 w, 742 m$. 
Anal. calcd for $\mathrm{C}_{17} \mathrm{H}_{14} \mathrm{O}_{3} \mathrm{~S}$ (298.37): $\mathrm{C}$ 68.43, H 4.74, S 10.74; found: C 68.67, H 4.98, S 10.91 .

Methyl 4-phenyl-6-(thiophen-2-yl)-4H-thiopyran-3-carboxylate (9k)

Orange oil; yield (method B): $222.9 \mathrm{mg}(71 \%) .{ }^{1} \mathrm{H} \mathrm{NMR}\left(600 \mathrm{MHz}, \mathrm{CDCl}_{3}\right): 3.70(\mathrm{~s}, 3 \mathrm{H}$, $\left.\mathrm{OCH}_{3}\right), 4.86(d, J=6.6 \mathrm{~Hz}, 1 \mathrm{H}), 6.27(d, J=6.6 \mathrm{~Hz}, 1 \mathrm{H}), 6.99(d d, J=5.4,3.6 \mathrm{~Hz}, 1 \mathrm{H})$, 7.15-7.16 (m, 1H), 7.22-7.24 ( $m, 2 \mathrm{H}), 7.31(t, J=7.2 \mathrm{~Hz}, 2 \mathrm{H}), 7.41(d, J=7.2 \mathrm{~Hz}, 2 \mathrm{H}), 7.72$ $(s, 1 \mathrm{H}) .{ }^{13} \mathrm{C}$ NMR $\left(150 \mathrm{MHz}, \mathrm{CDCl}_{3}\right): 41.2(\mathrm{C}(4)), 52.1\left(\mathrm{OCH}_{3}\right), 123.2,124.4,140.7,143.7$ (4 $\left.\mathrm{C}\left(\mathrm{sp}^{2}\right)\right), 121.4,124.7,125.3,127.3,127.6,128.2,128.9,132.1\left(10 \mathrm{CH}\left(\mathrm{sp}^{2}\right)\right), 165.1(\mathrm{C}=\mathrm{O}) . \quad \mathrm{IR}$ (KBr): $3025 w, 2949 w, 1711 v s\left(v_{\mathrm{C}=0}\right), 1587 m, 1423 m, 1230 s, 1036 m, 761 v s$. Anal. calcd for $\mathrm{C}_{17} \mathrm{H}_{14} \mathrm{O}_{2} \mathrm{~S}_{2}$ (314.43): C 64.93, H 4.50, S 20.39; found: C 64.95, H 4.77, S 20.14.

Methyl 6-(furan-2-yl)-4-phenyl-4H-thiopyran-3-carboxylate (91)

Yield: $6 \%$ (method A). The compound was identified only on the basis of the ${ }^{1} \mathrm{H}$ NMR spectra and could not be isolated in pure form. The yield was calculated based on the ${ }^{1} \mathrm{H}$ NMR spectra of the crude mixture using a weighed amount of 1,1,2,2-tetrachloroethane as a standard. ${ }^{1} \mathrm{H}$ NMR $\left(600 \mathrm{MHz}, \mathrm{CDCl}_{3}\right): 3.69\left(s, 3 \mathrm{H}, \mathrm{OCH}_{3}\right), 4.84(d, J=6.6 \mathrm{~Hz}, 1 \mathrm{H}), 6.39$ $6.41(m, 2 \mathrm{H}), 6.44(d, J=6 \mathrm{~Hz}, 1 \mathrm{H}), 7.28-7.31(m, 3 \mathrm{H}), 7.36-7.39(m, 3 \mathrm{H}), 7.71(s, 1 \mathrm{H})$. MS (ESI): $m / z(\%)=321\left(100,[\mathrm{M}+\mathrm{Na}]^{+}\right)$.

Methyl 4-phenyl-6-(selenophen-2-yl)-4H-thiopyran-3-carboxylate (9m)

Orange oil; yield (method B): $216.4 \mathrm{mg}(60 \%) .{ }^{1} \mathrm{H}$ NMR (600 MHz, $\left.\mathrm{CDCl}_{3}\right): 3.70(\mathrm{~s}, 3 \mathrm{H}$, $\left.\mathrm{OCH}_{3}\right), 4.85(d, J=6.6 \mathrm{~Hz}, 1 \mathrm{H}), 6.22(d, J=6.6 \mathrm{~Hz}, 1 \mathrm{H}), 7.22-7.25(m, 2 \mathrm{H}), 7.30-7.33$ (m, $3 \mathrm{H}), 7.40-7.42(m, 2 \mathrm{H}), 7.70(s, 1 \mathrm{H}), 7.90(d d, J=6.0,1.2 \mathrm{~Hz}, 1 \mathrm{H}) .{ }^{13} \mathrm{C}$ NMR $(150 \mathrm{MHz}$, $\left.\mathrm{CDCl}_{3}\right)$ : $41.4(\mathrm{C}(4)), 52.0\left(\mathrm{OCH}_{3}\right), 124.5,125.2,143.6,146.5\left(4 \mathrm{C}\left(\mathrm{sp}^{2}\right)\right), 122.0,126.9,127.3$, 128.2, 128.9, 130.1, 130.5, $132.2\left(10 \mathrm{CH}\left(\mathrm{sp}^{2}\right)\right), 165.1(\mathrm{C}=\mathrm{O})$. $1710 v s\left(v_{\mathrm{C}=0}\right), 1587 w, 1432 m, 1226 s, 1040 m, 742 m$. Anal. calcd for $\mathrm{C}_{17} \mathrm{H}_{14} \mathrm{O}_{2} \mathrm{SeS}$ (361.33): C 56.50, H 3.91, S 8.87; found: C 56.41, H 4.25, S 8.57.

Methyl 4,6-bis(thiophen-2-yl)-4H-thiopyran (9n) 
Orange oil; yield (method A): $310.4 \mathrm{mg}(97 \%) .{ }^{1} \mathrm{H}$ NMR $\left(600 \mathrm{MHz}, \mathrm{CDCl}_{3}\right): 3.78(\mathrm{~s}, 3 \mathrm{H}$, $\left.\mathrm{OCH}_{3}\right), 5.22(d, J=6.6 \mathrm{~Hz}, 1 \mathrm{H}), 6.34(d, J=6.6 \mathrm{~Hz}, 1 \mathrm{H}), 6.92-6.93(m, 1 \mathrm{H}), 6.98(d, J=3.6$ $\mathrm{Hz}, 1 \mathrm{H}), 7.01-7.02(m, 1 \mathrm{H}), 7.16(d d, J=5.4,1.2 \mathrm{~Hz}, 1 \mathrm{H}), 7.20(d d, J=53.6,0.6 \mathrm{~Hz}, 1 \mathrm{H})$, 7.25-7.26 (m, 1H), $7.68(\mathrm{~s}, 1 \mathrm{H}) .{ }^{13} \mathrm{C} \mathrm{NMR}\left(150 \mathrm{MHz}, \mathrm{CDCl}_{3}\right): 35.7(\mathrm{C}(4)), 52.2\left(\mathrm{OCH}_{3}\right)$, 124.3, 125.0, 140.3, $146.2\left(4 \mathrm{C}\left(\mathrm{sp}^{2}\right)\right), 120.0,124.76,124.84,125.1,125.7,127.0,127.7,132.4$ $\left(8 \mathrm{CH}\left(\mathrm{sp}^{2}\right)\right), 164.8(\mathrm{C}=\mathrm{O})$. IR (film): 3041w, 2952w, 1706vs $\left(v_{\mathrm{C}=0}\right), 1581 \mathrm{~m}, 1432 \mathrm{~m}, 1242 s$, $1226 s, 1024 m, 755 w, 701 v s$. Anal. calcd for $\mathrm{C}_{15} \mathrm{H}_{12} \mathrm{O}_{2} \mathrm{~S}_{3}$ (320.45): C 56.22, H 3.78, S 30.01; found: C 56.37, H 3.91, S 29.88.

\section{Disclosure statement}

No potential conflict of interest was reported by the authors.

\section{Funding}

This work was supported by the National Science Center (Cracow, Poland) within the project Maestro [Grant Number: Dec-2012/06/A/ST5/00219].

\section{References}

[1] Tietze LF. Domino reactions in organic synthesis. Chem Rev. 1996;96:115-136.

[2] Tietze, LF, Kettschau G. Hetero-Diels-Alder reactions in organic chemistry. Top Curr Chem. 1997;189:1-120.

[3] Eschenbrenner-Lux V, Kumar K, Waldmann H. The asymmetric hetero-Diels-Alder reaction in the synthesis of biologically relevant compounds. Angew Chem Int Ed. 2014;53:11146-11157.

[4] Rohr U, Schatz J, Sauer J. Thio- and selenocarbonyl compounds as 'superdienophiles' in [4+2] cycloadditions. Eur J Org Chem. 1998;2875-2883.

[5] Bogdanowicz-Szwed K, Budzowski A. Synthesis of polyfunctionalized thiopyrans via hetero-Diels-Alder reactions. Wiad Chem. 2003;57:435-457 (PL ISSN 0043-5104).

[6] Deryabina TG, Demina MA, Belskaya NP, Bakulev VA. Reaction of 3-aryl-2cyanothioacrylamides with dimethyl acetylenecarboxylate, methyl propiolate, and $N$ phenylmaleimide. Russ Chem Bull. 2005;54:2880-2889.

[7] Zelisko N, Atamanyuk D, Vasylenko O, Bryhas A, Matiychuk V, Gzella A, Lesyk R. Crotonic, cynnamic, and propiolic acids motifs in the synthesis of thiopyrano[2,3$d][1,3]$ thiazoles via hetero-Diels-Alder reaction and related tandem processes. Tetrahedron. 2014;70:720-729. 
[8] Zelisko N, Atamanyuk D, Ostapiuk Y, Bryhas A, Matiychuk V, Gzella A, Lesyk R. Synthesis of fused thiopyrano[2,3-d][1,3]thiazoles via hetero-Diels-Alder reaction related tandem and domino processes. Tetrahedron. 2015;71:9501-9508.

[9] Lozynskyi A, Zimenkovsky B, Gzella AK, Lesyk R. Arylidene pyruvic acids motif in the synthesis of new $2 H, 5 H$-chromeno[4',3':4,5] thiopyrano[2,3- $d$ ] thiazoles via tandem hetero-Diels-Alder reaction. Synth Commun. 2015;45:2266-2270.

[10] Lozynskyi A, Zimenkovsky B, Karkhut A, Polovkovych S, Gzella AK, Lesyk R. Application of the $2(5 H)$ furanone motif in the synthesis of new thiopyrano[2,3d] thiazoles via the hetero-Diels-Alder reaction and related tandem processes. Tetrahedron Lett. 2016;57:3318-3321.

[11] Kamila S, Biehl E. Preparation of benzothiopyrano[2,3-b]indoles by the reaction of 1,3-dihydroindole-2-thiones with certain dienophiles. Heterocycles. 2004;63:27852795.

[12] Majumdar KC, Taher A, Ponra S. Catalyst-free regioselective synthesis of benzopyran-annulated thiopyrano[2,3- $b]$ thiochromen-5(4H)-one derivatives by domino-Knoevenagel-hetero-Diels-Alder reaction of terminal alkynes with 4-hydroxy dithiocoumarin in aqueous medium. Tetrahedron Lett. 2010;51:2297-2230.

[13] Ohmura H, Motoki S. Cycloaddition reactions of heteroaromatic thioketones with maleic anhydride, norbornene, acrylonitrile, styrene, and 2-dichloroacrylonitrile. Bull Chem Soc Jpn. 1984;57:1131-1137.

[14] Mlostoń G, Grzelak P, Mikina M, Linden A, Heimgartner H. Hetero-Diels-Alder reactions of hetaryl and aryl thioketones with acetylenic dienophiles. Beilstein J Org Chem. 2015;11:576-582.

[15] Karakasa T, Motoki S. Chemistry of $\alpha, \beta$-unsaturated thione dimers. 1. Preparation of $\alpha, \beta$-unsaturated thione dimers and thermolysis of these dimers in the presence of acrylonitrile or acrylamide. J Org Chem. 1978;43:4147-4150.

[16] Karakasa T, Motoki S. Chemistry of $\alpha, \beta$-unsaturated thione dimers. 2. Reactions of thiochalcones and 2-arylidene-1-thiotetralones with some olefins and the parent ketones of the thiones. J Org Chem. 1979;44:4151-4155

[17] Tanaka H, Motoki S. Chemistry of 1,2-thiaphosphole 2-sulfides. III. Cycloaddition reactions with some acetylenic dienophiles and generation of phosphorin 1-sulfides. Bull Chem Soc Jpn. 1986;59:2047-2049.

[18] Nishio T, Nakajima N, Omote Y. Photochemical reactions of $\beta$-aminovinyl aryl thioketones. J Heterocycl Chem. 1980;17:405-406. 
[19] Rasmussen JB, Shabana R, Lawesson S.-O. Enamine chemistry - XXIII: The [4+2] cycloaddition reactions between enaminothiones and electrophilic olefins and acetylenes. Tetrahedron. 1981;37:3693-3698.

[20] Saito T, Fujii H, Hayashibe S, Matsushita T, Kato H, Kobayashi K. Uncatalised (termal) and Lewis acid-promoted asymmetric hetero-Diels-Alder reaction of 1thiabuta-1,3-dienes (thiochalcones) with di-(-)-menthyl fumarate. Configuration determination by X-ray crystallographic analysis of $(2 S, 3 R, 4 R)-(+)-2,3-b i s[(-)-$ menthoxycarbonyl]-4,6-diphenyl-3,4-dihydro- $2 H$-thiopyran and conversion of cycloadducts into optically pure diols. J Chem Soc Perkin Trans 1. 1996;1897-1903.

[21] In Organikum; Organisch-chemisches Grundpraktikum, E. Fanghänel, Ed., 23. Edition, Wiley-VCH Verlag GmbH, Weinheim 2009, p. 527.

[22] Yoshisato E, Ryang M, Tsutsumi S. Reaction of iodobenzene and nickel carbonyl in the presence of olefins. J Org Chem. 1969;34:1500-1502.

[23] Lown JW, Matsumoto K. Synthesis and cycloaddition reactions of 2-(2thienyl)aziridines. Can J Chem. 1970;48:2215-2226.

[24] Powell P, Sosabowski MH. Preparation and reactions of some 2-thienyl and 3thienylpyridazinones and -pyridazines. J Chem Res (S). 1995;306-307; J Chem Res (M). 1995;1840-1852.

[25] El-Rayyes NR. Heterocycles. Part I. A new route to the synthesis of substituted 2aminopyrimidines. J Heterocycl Chem, 1982; 19: 415-41.

[26] Budhiraja A, Kadian K, Kaur M, Aggarwal V, Garg A, Sapra S, Nepali K, Suri OP, Dhar KL. Synthesis and biological evaluation of naphthalene, furan and pyrrole based chalcones as cytotoxic and antimicrobial agents. Med Chem Res. 2012;21:2133-2140.

[27] Umezawa S. Synthetische Versuche in der Selenophen-Gruppe. IV. Einführung von Seitenketten in den Selenophen-Kern. Bull Chem Soc Jpn. 1939;14:155-161. 